\title{
The effect of population density on shoot morphology of herbs in relation to light capture by leaves
}

\author{
Toshio Sekimura ${ }^{\mathrm{a}, *}$, Tiina Roose ${ }^{\mathrm{b}}$, Bo $\mathrm{Li}^{\mathrm{c}, 1}$, Philip K. Maini ${ }^{\mathrm{b}}$, \\ Jun-ichirou Suzuki ${ }^{\mathrm{c}}$, Toshihiko Hara ${ }^{\mathrm{c}}$ \\ ${ }^{a}$ College of Engineering, Chubu University, Kasugai, Aichi 487-8501, Japan

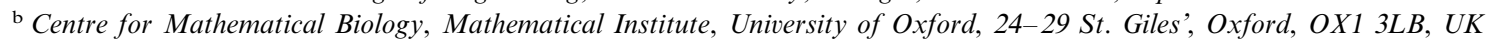 \\ ${ }^{\mathrm{c}}$ The Institute of Low Temperature Science, Hokkaido University, Sapporo 060-0819, Japan
}

Accepted 8 November 1999

\begin{abstract}
Plants change their shapes, depending on their environment, for example, plant height increases with increasing population density. We examined the density-dependent plasticity in shoot morphology of herbs by analysing a mathematical model which identifies a number of key factors that influence shoot morphology, namely (i) solar radiation captured by leaves; (ii) shading from neighbouring plants; and (iii) utilisation efficiency of resource by leaves, stems and veins. An optimisation theory was used to obtain optimal shoot morphology in relation to maximal light capture by leaves, under trade-offs of resource partition among organs. We first evaluated the solar radiation flux per unit leaf area per day for different shoot forms. Our model predicts that the optimal internodal length of the stem that brings about the maximal light capture by leaves increases with plant population density, and this is consistent with experimental data. Moreover, our simple model can also be extended to explain the morphological plasticity in other herbs (i.e. stemless plants) that are different from our model plants with a stem. These findings illustrate how optimisation theory can be used for the analysis of plasticity in shoot morphology of plants in response to environmental changes, as well as the analysis of diversity in morphology. (C) 2000 Elsevier Science B.V. All rights reserved.
\end{abstract}

Keywords: Density effect; Inter-plant competition; Internodal length; Morphological plasticity; Optimisation theory

\footnotetext{
* Corresponding author. Tel.: +81-568-51-1111, ext. 2602.

E-mail address: sekimura@isc.chubu.ac.jp (T. Sekimura)

${ }^{1}$ Present address: Upland Weed Laboratory, National Agriculture Research Centre, Kan-nomdai 3-1-1, Tsukuba 3058666, Japan.
}

\section{Introduction}

Plants exhibit great morphological plasticity in their response to the environment such as the number of neighbouring plants (i.e. population density). Plant height, for instance, increases (relative to biomass, stem diameter and leaf area) as population density increases (e.g. Hara, 1984; 
Sekiyama et al., 1984a,b; Schmitt and Wulff, 1993). Such plasticity in plants was previously explained by game theory (e.g. Givnish, 1982; Iwasa et al., 1984; Givnish, 1986; Sakai, 1991). Increasing experimental evidence also shows that such plastic responses of plants to competitive environments are physiologically phytochrome-mediated and can confer plants' ability to forage for light, and thus are adaptive (see reviews by Schmitt and Wulff, 1993 and Smith, 1994).

In our preceding papers (Sekimura, 1993, 1995, 1997), we quantitatively estimated the solar radiation flux captured by leaves of model plants and discussed the diversity in shoot morphology of herbs. In this paper, we consider the density effect on shoot morphology of herbs through numerical calculations of the flux which take account of both self-shading of a model plant and 'alien'shading from neighbouring plants. Optimisation theory is used to obtain optimal shoot morphology in relation to maximal light capture under the trade-offs of resource partition among leaves, stems and veins. The model is formulated and analysed in Section 2, and the optimisation results are presented in Section 3. Section 4 contains a full discussion of the significance of our results.

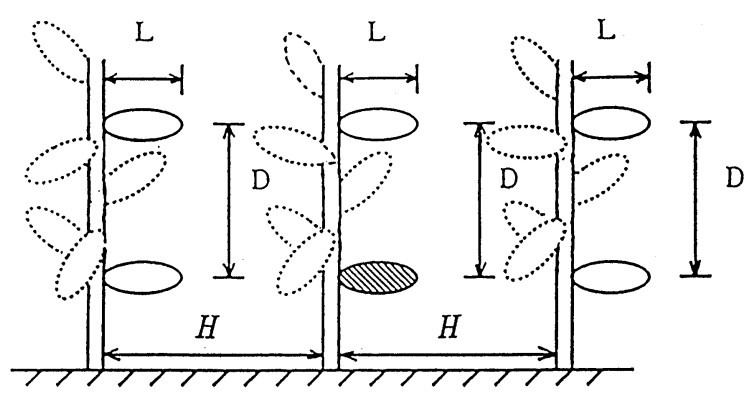

Fig. 1. Model plants with vertical stems standing at regular intervals on a straight line. The parameters $L, D$, and $H$ are leaf lamina length, the internodal length of the stem between two leaves at the same phyllotactic position, and the distance between two neighbouring plants, respectively.

\section{The model}

\subsection{Basic assumptions in model simulations}

Model plants have a single vertical stem with horizontally oriented leaves and stand at regular intervals along a straight line (Fig. 1).

We assume that the sun rises in the east and sets in the west after passing overhead within a certain timeframe (e.g. $12 \mathrm{~h}$ ).

Light shading for a leaf in question comes from both the upper leaf at the same phyllotactic position of the plant and upper leaves at the same position of both its right and left neighbouring plants. When the distance between two neighbouring plants is very small, the leaf overlap is treated in such a way as to exclude double counting of light shading (see Section 2.2.2 for details). The internodal length of the stem and leaf shape are designed to maximise

1. the flux of solar radiation captured by leaves and

2. the utilisation efficiency of resources.

Leaf area and internodal length of a plant are constrained by the trade-offs of resource partition among leaves, stems, and veins, so as to maximise the solar radiation flux per leaf per day.

\subsection{Solar radiation flux captured by leaves}

The solar radiation flux per leaf per day, $N$, is evaluated by the following equation:

$$
N=\int_{t_{0}}^{t_{1}} I_{0}\left(S-S_{0}^{\prime}\right) \sin \theta \mathrm{d} t
$$

where $t_{0}$ and $t_{1}$ are the times at which the sun rises and sets, respectively; $I_{0}$ is the incident solar flux per unit leaf area per unit time. $\theta$ is the elevation angle of the sun measured from the plant, and $S$ is the surface area of a leaf in question, which is easily calculated if the leaf outline is given by a mathematical function (Eq. (A1), Appendix A). $S_{\theta}^{\prime}$ is the shaded area on the leaf at sun elevation $\theta$, which is a function of time $t$, distance $H$ between two neighbouring stems, internodal length $D$ of the stem between two leaves at the same phyllotactic position,

$S_{\theta}^{\prime}=S^{\prime}(t, H, D)$ 


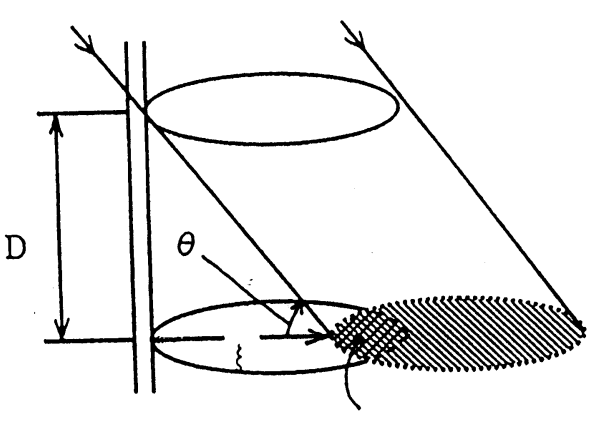

Shaded area intercepted by the upper leaf

Fig. 2. Self-shading of light for a leaf in question from the upper leaf at the same phyllotactic position. $\theta$ is the elevation angle of the sun measured from the plant. $\xi$ is the distance between the stem and the tip of the shadow on the leaf. $D$ is the internodal distance of the stem between two leaves at the same position.

(a)

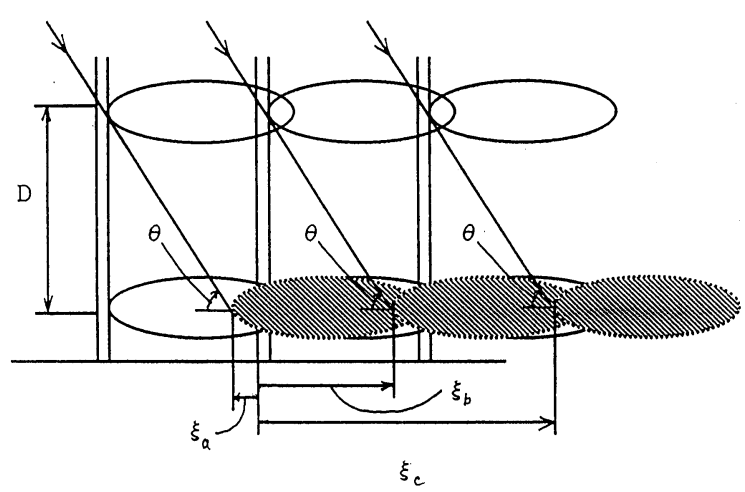

Fig. 3. Light shading from leaves including both right and left neighbouring plants. Symbols as in Fig. 2.

Generally, $S_{\theta}^{\prime}$ is not a simple mathematical function even if the leaf outline is given by a simple mathematical function. We therefore consider shading on a leaf for the following cases.

\subsubsection{Light shading from an upper leaf at the same phyllotactic position}

We firstly present the basic idea of how to calculate the solar radiation flux on a leaf lamina. Self-shading of light for the lower leaf in question is assumed to come from an upper leaf at the same phyllotactic position (Fig. 2). The position of the tip of the shadow cast by the upper leaf on the lower leaf measured from the stem, $\xi$, is given by

$\xi=D / \tan (\theta)$

where $\theta$ is the elevation angle of the sun measured from the plant. During the time/angle interval in which the condition $0 \leq \xi \leq L$ (leaf lamina length) holds, the leaf is shaded by the upper leaf (see Appendix B for more details).

\subsubsection{Light shading from upper leaves of three plants including both right and left neighbours}

We consider light shading from three upper leaves of three plants including both its right and left neighbouring plants. The shading area to be estimated is the area on the lower leaf of the middle plant (b) of the three plants (a), (b), (c) in Fig. 3, which stand at regular intervals $H$ on a straight line. We have assumed the origin of $x$ co-ordinates to be the stem position of the middle plant (b). As shown in Fig. 3, $\xi_{\mathrm{b}}$ is the position of the tip of the shadow of the upper leaf of plant (b) measured from the origin. We express the position of the tip of the shadow of the upper leaf of plant (a) on the lower leaf's plane of plant (b), by $\xi_{\mathrm{a}}=H+D / \tan (\theta) . \xi_{\mathrm{c}}$ represents the position of the tip of the shadow from the upper leaf of plant (c) on the lower leaf's plane of plant (b):

$\xi_{\mathrm{c}}=H+D / \tan (\theta)$.

The shading area $S_{\theta}^{\prime}$ on the lower leaf of plant (b) is expressed as follows:

$S_{\theta}^{\prime}=S_{0}^{\prime}+S_{\mathrm{a}}^{\prime}+S_{\mathrm{b}}^{\prime}+S_{\mathrm{c}}^{\prime}-S_{\mathrm{ab}}^{\prime}-S_{0 \mathrm{abc}}^{\prime}-S_{\mathrm{bc}}^{\prime}$

where $S_{0}^{\prime}$ is the shade from the lower leaf of plant (a); $S_{\mathrm{a}}^{\prime}, S_{\mathrm{b}}^{\prime}, S_{\mathrm{c}}^{\prime}$ are shades from the upper leaves of plants (a), (b), (c), respectively; $S_{\mathrm{ab}}^{\prime}$ is the intersection of shades $S_{\mathrm{a}}^{\prime}$ and $S_{\mathrm{b}}^{\prime} ; S_{0 \mathrm{abc}}^{\prime}$ is the intersection of shades $S_{0}^{\prime}, S_{\mathrm{a}}^{\prime}, S_{\mathrm{b}}^{\prime}$, and $S_{\mathrm{c}}^{\prime} ; S_{\mathrm{bc}}^{\prime}$ is the intersection of shades $S_{\mathrm{b}}^{\prime}$ and $S_{\mathrm{c}}^{\prime}$. When overlap between lower leaves of neighbouring plants occurs, that is, when the condition $H<L$ holds, it is reasonable to assume that the lower leaf of plant (a) is placed on the lower leaf of the middle plant (b), whereas the lower leaf of plant (c) is placed under the middle one. When no overlap between leaves occurs, Eq. (3) can be simplified to the following expression (see Appendix B for more details):

$S_{\theta}^{\prime}=S_{\mathrm{a}}^{\prime}+S_{\mathrm{b}}^{\prime}+S_{\mathrm{c}}^{\prime}$ 


\subsection{Optimisation theory}

We consider the trade-offs of resource partition among leaves, stem, and veins of a plant, under the following conditions:

$X+Y+Z=R$

$X=\sigma S(L, W) ; Y=\rho_{1} V_{1}\left(D, r_{1}\right) ;$ and $Z=\rho_{2} V_{2}\left(L, r_{2}\right)$

where $X, Y$ and $Z$ are the fractions of resource $R$ allocated to leaf lamina, stem, and vein, respectively. Parameters $\sigma, \rho_{1}$, and $\rho_{2}$ represent actual partition coefficients of resource to leaf lamina, stem, and vein, respectively. $S$ denotes the leaf area in question, which is a function of leaf lamina length $L$ and width $W$ (cf. Eq. (A2)); $V_{1}$ is the volume of the stem between two leaf laminae located at the same position, and is a function of the internodal length $D$ and the stem radius $r_{1} ; V_{2}$ is the volume of a main vein, and is assumed to be a function of leaf lamina length $L$ and radius $r_{2}$ of the vein. The units of $\sigma S, \rho_{1} V_{1}$ and $\rho_{2} V_{2}$ are taken to be the same as that of the resource $R$, whatever the unit of resource is.

When the amount of total resource is constant, that of the resource a plant can obtain, $R$, is assumed to be a function of plant population density, being inversely correlated with density. When plants are placed at regular intervals of distance $H$, the population density is the reciprocal of $H$. Hence we assume that the resource $R$ is proportional to the interval $H$. For simplicity, we assume that the resource, $R$, is constant when the distance between two plants is more than twice the leaf lamina length $L$.

\section{Results}

For convenience, we normalise distance $(H)$, internodal length $(D)$, and leaf lamina width $(W)$ by leaf lamina length $L$ to obtain $\bar{H}(=H / L)$, $\bar{D}(=D / L)$, and $\bar{W}(=W / L)$. We also normalize the resource $(R)$ by the parameter $\sigma$ to give $\bar{R}(=R / \sigma)$.

\subsection{Flux including shading from neighbouring plants}

The flux per unit leaf area per day is shown in Fig. 4, in which both self-shading of the plant in

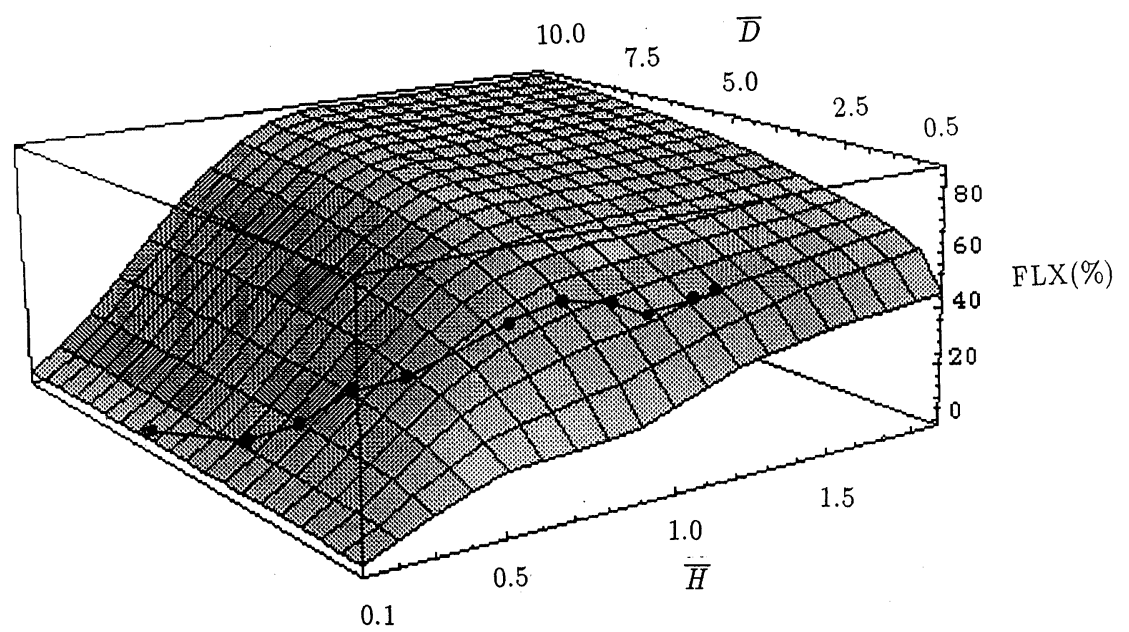

Fig. 4. Solar radiation flux per unit leaf area per day, in which self-shading is included in the estimation as well as shading from both neighbouring plants, one on each side. Values of the flux, $F L X(\%)$, are normalised by dividing them by the flux value for a non-shaded leaf. $\bar{H}(=H / L)$ is the normalised distance between two neighbouring plants divided by leaf lamina length $L . \bar{D}(=D / L)$ is the normalised internodal length of the stem of a single plant, divided by $L$. The optimal pathway on the surface of the flux is also given. The closed circles connected by solid lines correspond to the case of leaf lamina width $\bar{W}=0.30$. 


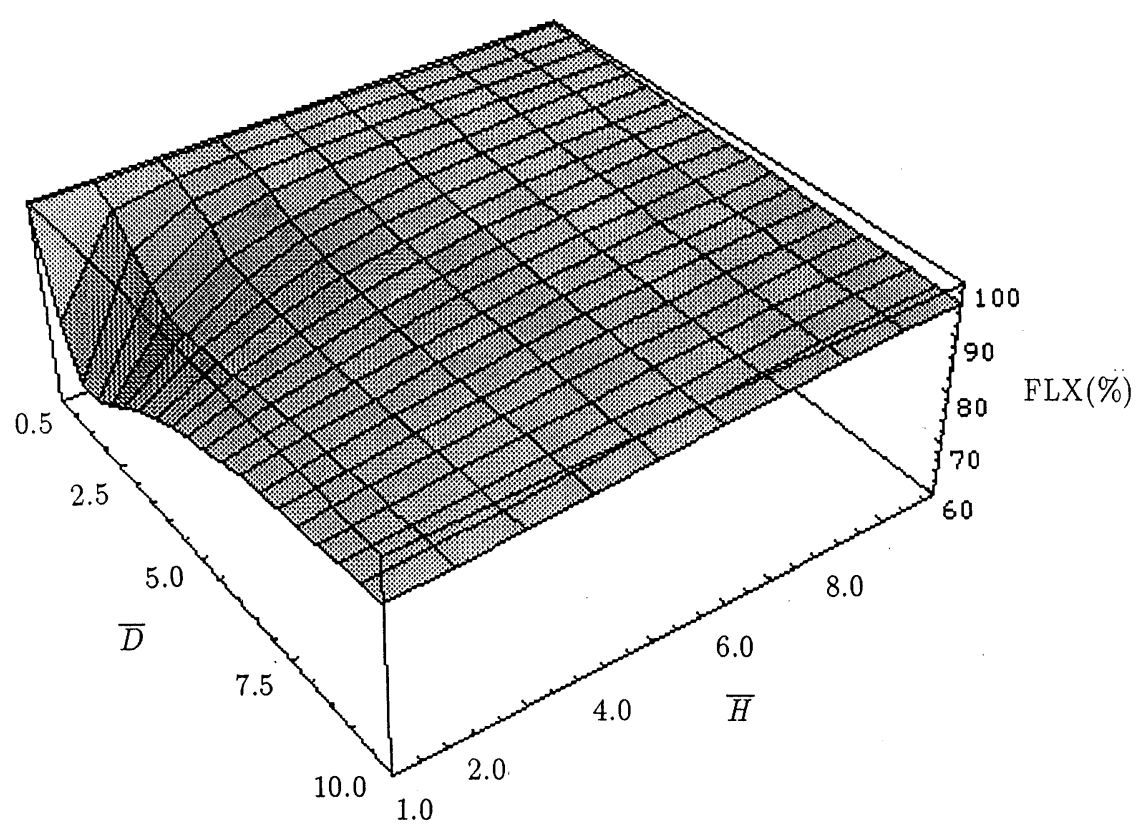

Fig. 5. Solar radiation flux per unit leaf area per day, in which only light shading from both the right and the left neighbours is estimated without self-shading of the plant. Values of the flux $F L X(\%)$ are normalised by dividing them by the flux value for a non-shaded leaf. $\bar{H}(=H / L)$ is the normalised distance between two neighbouring plants divided by leaf lamina length $L . \bar{D}(=D / L)$ is the normalised internodal length of the stem of a single plant, divided by $L$.

question and alien-shading from neighbouring plants, one on either side, are taken into account. In Fig. 5, we show the flux from the neighbouring plants in the case of no self-shading. We normalised the values of the flux, $F L X(\%)$, by dividing them by the flux value for a non-shaded leaf. It can be clearly seen that the flux, FLX $(\%)$, decreases rapidly over the range $1.0-2.5$ of the internodal distance $\bar{D}$ and the range 2.0-3.0 for the distance between two plants $\bar{H}$. Our analysis shows that the decrease in flux reflects the geometrical coincidence between leaf position on the stem and stem positions of two neighbouring plants, in relation to light shading. This affects the change in the flux in Fig. 4 and Fig. 6, where both self-shading and alien-shading from both side plants are considered.

\subsection{Flux under the trade-offs of resource partition among the organs}

The relationship between the flux $F L X(\%), \bar{H}$ and $\bar{W}$ is shown in Fig. 6. The flux $\operatorname{FLX}(\%)$ was calculated according to optimisation theory under the trade-offs of resource partition, in which selfshading, together with alien-shading from neighbouring plants is considered. It can be clearly seen from Fig. 6 that a rapid decrease in the flux $F L X(\%)$ from 60 to $0 \%$ occurs over a small range of the inter-plant distance around $\bar{H}=0.5$. The value $\bar{H}=0.5$ is the distance between two neighbouring plants at which two leaves at the same position of both plants overlap by half. The decrease in the flux results in a corresponding increase in the internodal length $\bar{D}$ between two leaves of the plant as a theoretical result of the assumption that the flux per leaf per day is to be maximised (see Fig. 7 in the following section).

\subsection{Relationship between the optimal internodal length and plant population density}

Fig. 7 shows that the optimal internodal length, $\bar{D}$, decreases with an increase in the distance between two neighbouring plants, $\bar{H}$, with respect to the maximal light capture constrained by con- 


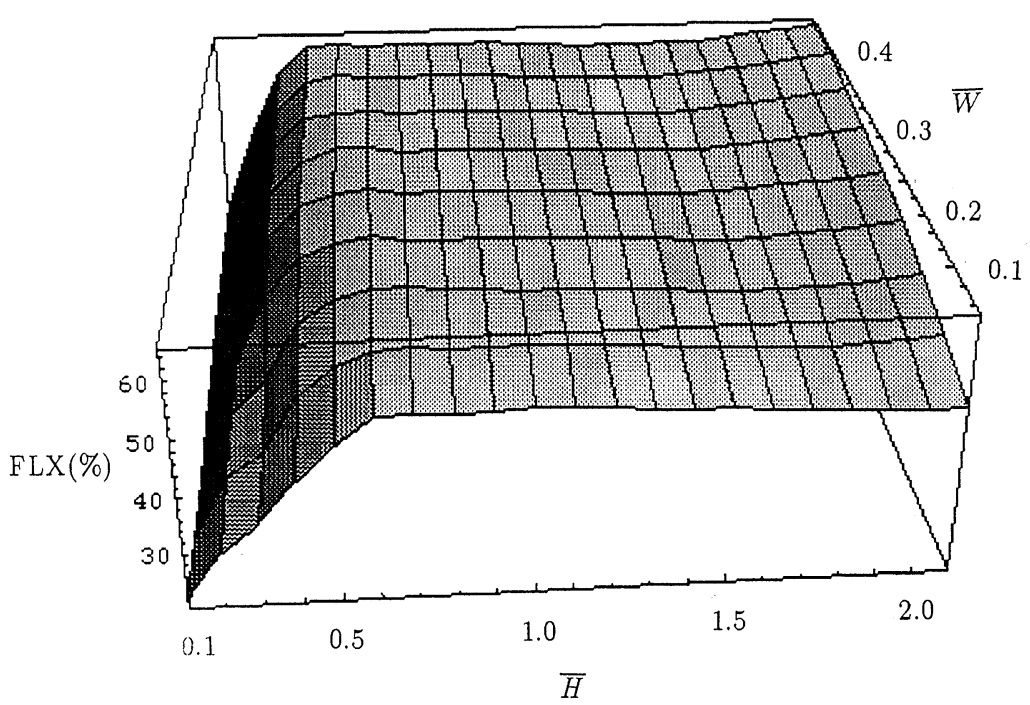

Fig. 6. Solar radiation flux per unit leaf area per day calculated by using optimisation theory, under trade-offs of resource partitionning among leaves, stems and veins. Light shading calculations include self-shading of the plant in question and shading from both neighbouring plants. Values of the flux, $F L X(\%)$, are normalised by dividing them by the flux value for a non-shaded leaf. $\bar{H}(=H / L)$ is the normalised distance between two neighbouring plants divided by leaf lamina length $L$. $\bar{W}(=W / L)$ is the normalised leaf lamina width divided by $L$.

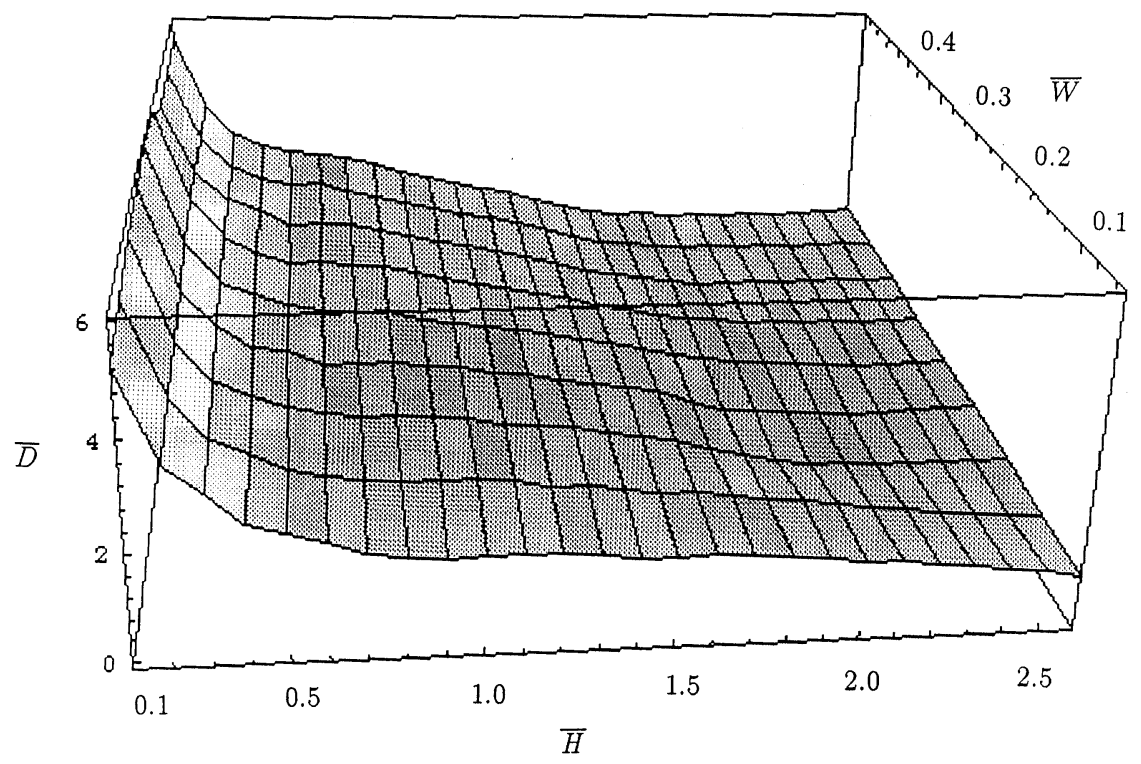

Fig. 7. Optimal internodal length $\bar{D}$ against the distance $\bar{H}$ between two neighbouring plants with leaf lamina width $\bar{W}$. $\bar{D}, \bar{H}$ and $\bar{W}$ are normalised as in Figs. 4-6. This figure corresponds to Fig. 6 because both the figures were calculated simultaneously with the same parameter values (i.e. leaf lamina width $\bar{W}$ and resource value $\bar{R}$ ). 
Table 1

Relationship between plant population density and internodal length of the main stem in soybean (G. max variety Tamanishiki) populations (recalculated from Sekiyama et al., 1984a)

\begin{tabular}{lcccc}
\hline Distance $H(\mathrm{~cm})^{\mathrm{a}}$ & Density (plants $\left.{ }^{-2}\right)$ & Stem length $(\mathrm{cm})^{\mathrm{b}}$ & Node number $^{\mathrm{c}}$ & ${\text { Node length }(\mathrm{cm})^{\mathrm{d}}}$ \\
\hline 28.9 & 12 & 66.5 & 18.5 & 3.59 \\
20.4 & 24 & 75.7 & 18.6 & 4.07 \\
14.4 & 48 & 99.6 & 18.5 & 5.38 \\
10.2 & 96 & 107.0 & 17.4 & 6.15 \\
7.22 & 192 & 111.1 & 16.3 & 6.82
\end{tabular}

${ }^{\text {a Distance }} H$ is the mean distance between two neighbouring plants, which is estimated by the following formula: $H(\mathrm{~cm})=100 /$ $\sqrt{\text { Density. }}$

${ }^{\mathrm{b}}$ Stem length is the mean plant height.

${ }^{\mathrm{c}}$ Node number is the mean number of nodes on the plant stem.

d Node length $D$ is the mean internodal length of the stem, which is obtained by dividing the stem length by the node number.

stant resource, namely, the optimal internodal length increases with plant population density. Obviously, Fig. 7 corresponds to Fig. 6, because both were calculated simultaneously with the same parameter values (i.e. leaf lamina width $\bar{W}$ and resource value $\bar{R}$ ). It can be seen from Fig. 7 that the internodal length $\bar{D}$ increases with leaf lamina width $\bar{W}$ when the distance $\bar{H}$ decreases to zero. Our predictions agree well with the experimental results shown in Table 1. Sekiyama et al. (1984a) carried out a field experiment with soybean (Glycine max variety Tamanishiki) at a range of densities to investigate effects of plant population density on yield. From Table 1 of Sekiyama et al. (1984a), we recalculated the distance between neighbouring plants and internodal lengths of the main stem at maturity (125 days after sowing). We see that the internodal length decreased with an increase in the distance between neighbouring plants, namely, the internodal length increased with plant population density (Table 1). This pattern held from about 50 days after sowing onwards (Sekiyama, Kuroiwa and Hara, unpublished data). Therefore, the theoretical predictions in Fig. 7 agree well with the experimental data in Table 1. Sekiyama et al. (1984b) repeated a similar yield-density experiment with two soybean varieties (G. max variety Tamanishiki and G. max variety Tamahomare) in 1983. Recalculation from their 1983 results also showed the same pattern as in Table 1. We can see the same relationship between internodal length and distance between neighbouring plants also in the experimen- tal results with sunflower (Helianthus annuus) of Kobayashi (1975).

We note that over the range of $\bar{H}<0.5$, a rapid increase in the optimal distance $\bar{D}$ can be seen, which is inversely related to the value of the flux in Fig. 6. It might be possible to define a critical distance ' $H c$ ' between two neighbouring plants, at which dramatic changes in both the flux and the internodal length of the stem are expected.

The optimal pathway on the surface of the flux is given also in Fig. 4 in the two parameter $(\bar{H}, \bar{D})$ domain for the case of Fig. 6 where the leaf lamina width $\bar{W}=0.30$.

\subsection{Relationship between optimal leaf length and plant population density}

Fig. 8 shows the relationship between the optimal leaf length, $L$, and the distance between neighbouring plants, $\bar{H}$, for a range of values of leaf lamina width $\bar{W}$. Fig. 8 corresponds to Fig. 6 and Fig. 7 because both the figures were calculated simultaneously with the same parameter values (i.e. leaf lamina width $\bar{W}$ and resource $\bar{R}$ ). Fig. 8 also shows that the optimal leaf length $L$ increases with decreasing leaf lamina width $\bar{W}$.

\section{Discussion}

We theoretically analysed the effect of population density on shoot morphology of herbs in relation to light capture by leaves. Using optimisa- 
tion theory, we were able to obtain the optimal shoot morphology which gives the maximal solar radiation flux per leaf per day under the trade-offs of resource partition among leaves, stems and veins.

Our model here focuses on the qualitative, rather than quantitative, nature of morphological changes within plants. We have shown, however, that this simple model can explain a variety of observed phenomena. The model framework developed in this paper can be further extended to investigate the effects of factors ignored in the present model.

For convenience, we assumed that the model plant has a single vertical stem. This is not, of course, always the case in reality. Our model for plants with an erect stem can also be applicable to herbs of different growth forms such as stemless or prostrate species. Generally, our model predicts that plants extend or equivalently invest more biomass in their supporting organs to maximise their light capture as plant population density increases. More specifically, while erect herbs increase their internodal length in response to the presence of neighbours or simulated competition (i.e. reduced red/far-red ratio), prostrate species

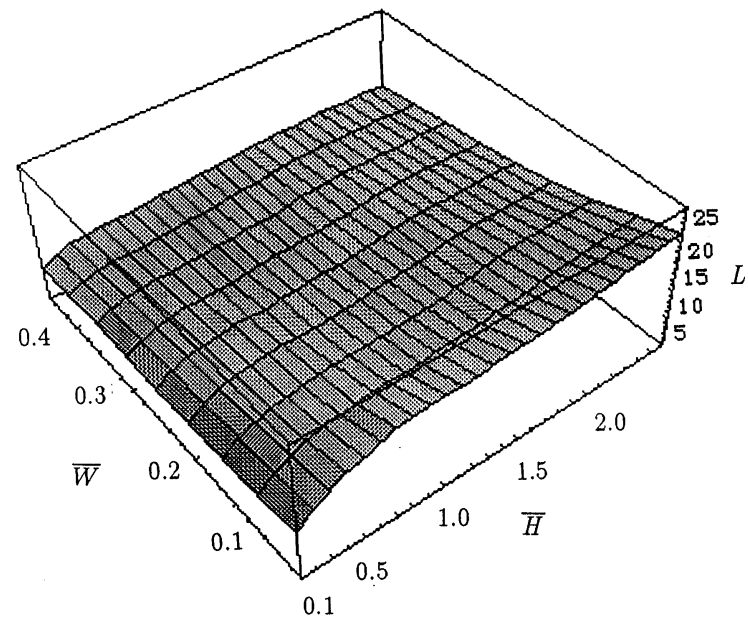

Fig. 8. Relationship between optimal leaf length $L$, the distance $\bar{H}$ between two neighbouring plants, and leaf lamina width $\bar{W}$. This figure corresponds to Figs. 6 and 7 because both the figures were calculated simultaneously with the same parameter values (i.e. leaf lamina width $\bar{W}$ and resource value $\bar{R}) . \bar{H}$ and $\bar{W}$ are normalised as in Fig. 4, Fig. 5 and Fig. 6. adjust the length of petioles and stemless herbs would invest more biomass in their supporting organs (e.g. extended hypocotyl or petiole). These are supported strongly by existing experimental evidence (e.g. Ballaré et al., 1988, 1990; Dale and Causton, 1992; Huber, 1997) and also by our experimental results obtained from two Brassica campestris varieties, cabbage and turnip (B. Li, J. Suzuki and T. Hara, unpublished data). They grew the two varieties at a wide range of densities and performed four consecutive harvests. It was observed that cabbage allocated a higher fraction of its biomass to its extended hypocotyl at higher densities, whilst turnip increased its biomass allocation to its petioles with increasing density (Fig. 9). The consequences of the morphological changes through increasing biomass allocation to the supporting organs are also to maximise their light capture. It is worth whilte to note that to increase the length of hypocotyl or petiole is the only way for these stemless plants to maximise their light capture. We can therefore draw a general conclusion that plants are able to find their ways of maximising their light capture in competitive environments at high densities by increasing either internodal length, hypocotyl or petiole.

As mentioned in the Introduction, morphological plasticity in response to competition for light has a physiological basis. Plants actually have information-acquiring systems that can detect and 'sense' the presence of neighbours by using phytochrome (Smith, 1982, 1994; Schmitt and Wulff, 1993; Aphalo and Ballare, 1995). When population density increases, mutual shading inevitably occurs, which leads to reduced light quantity and red/far-red ratio (an indicator of light quality). Phytochrome acts to detect mutual shading through the changed quality of natural radiation, and to redirect development appropriately (Holmes and Smith, 1975; Morgan and Smith, 1976; Smith, 1995). Plants can, through phytochrome-mediated photomorphogenesis, respond to both competitive (at high population density) and simulated-competition (through reduced red/ far-red ratio) environments by increasing stem extension (Morgan and Smith, 1976; Ballaré et al., 1990; Dale and Causton, 1992), internode elongation (Smith, 1982; Ballaré et al., 1988; Davis and Simmons, 1994; Huber, 1997) and petiole length 

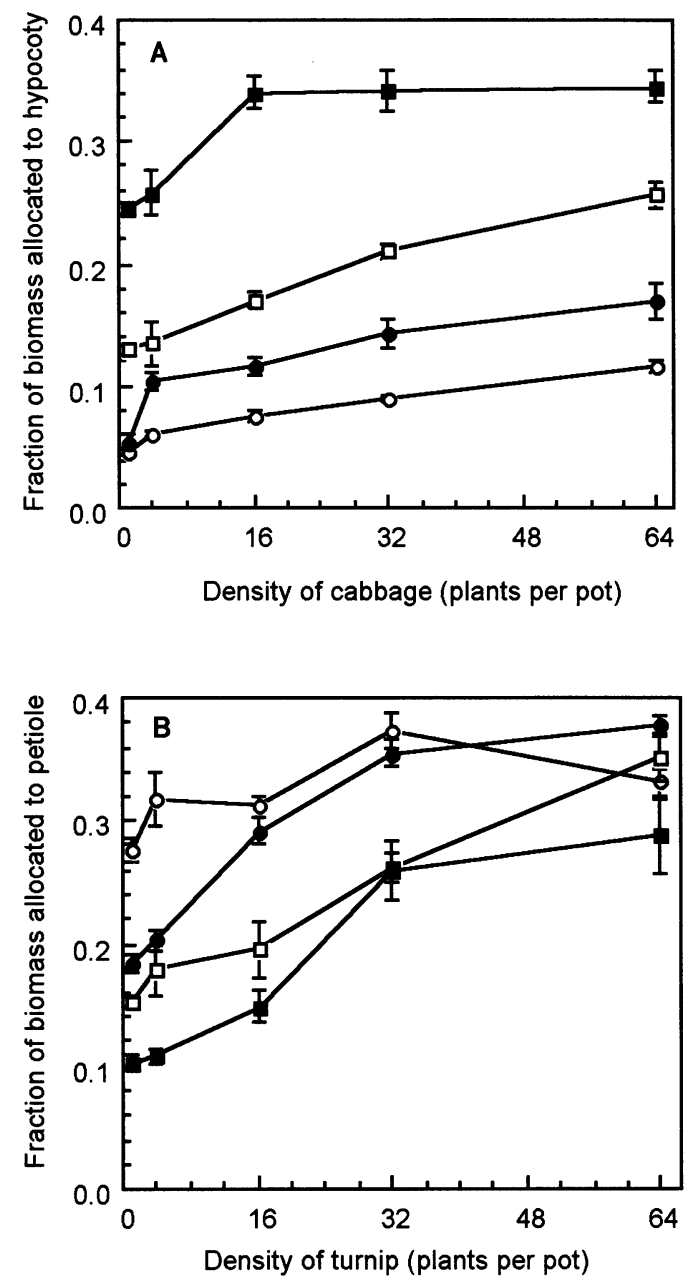

Fig. 9. Effects of plant population density on the fraction of total biomass allocated to the extended hypocotyl of cabbage (A) and petiole of turnip (B) at $30(\bigcirc), 60(\bullet), 90(\square)$ and 120 (ם) days after emergence. The vertical bars represent standard errors (from unpublished data by B. Li, J. Suzuki and T. Hara).

(Thompson and Harper, 1988; Dale and Causton, 1992; Huber, 1997). Therefore, a generalisation can be reached that plants can, in response to canopy shade due to competition at high population density, increase their potential for light capture by increasing vertical growth or horizontal spread (depending on their growth forms). Moreover, a suite of morphological traits conferring plants' ability to forage for light can also be expected in plants that are well adapted to longterm shaded habitats.
The plants in our model have a stem with horizontally oriented leaves, while leaves of real plants are attached to the stem with some leaf deflection angle. This assumption can be replaced by a more realistic assumption when we have systematic and accurate experimental data with which to compare model results.

In this study, we focused on the shoot morphology of a single plant. Other studies have focused on the global/overall pattern of plant communities. For example, it was reported that protonemal colonies of the moss Scopelophila cataractae show wave-like patterns in relation to light capture (Takenaka and Satake, 1991).

Plants change their shapes considerably to adapt themselves to changing environments. The phenotypic plasticity of plants and the rigidity of genes seem to generate and stabilise the diversity of plant forms. In order to fully understand this diversity, it is necessary to consider plants from a comprehensive view including ontogeny, genetics, ecology, evolution and physical conditions. Optimisation theory has been used mainly for modelling resource or ecosystem management (e.g. Van der Molen and Pintér, 1993; Straškraba, 1994; Håkanson, 1995; Tiwari et al., 1996; Williams, 1996). Our paper illustrates how optimisation theory can be used for the analysis of plasticity in shoot morphology of plants in response to environmental changes, as well as the analysis of diversity in morphology. Our model based on optimisation theory gives a basis for the study of the effects of environmental changes on plant morphology and plant community diversity in a local area and will be further extended to global terrestrial ecosystem models (cf. Jørgensen, 1997a,b; Müller, 1997) by incorporating latitudinal change in the sun elevation.

\section{Acknowledgements}

We thank Dr S. Sakai for valuable comments on the manuscript. This work was supported in part by a grant from the Research Institute of Science and Technology, Chubu University, Japan to TS, a grant from Hertford College, 
University of Oxford, UK to PKM and TS, and a grant of the Japan Society for the Promotion of Science Postdoctoral Fellowship to BL. TS acknowledges the Centre for Mathematical Biology, University of Oxford, for its hospitality. TR acknowledges the Centre for Mathematical Biology, University of Oxford, for its hospitality, and the Foreign and Commonwealth Office for a Marshall fellowship. Part of this work was carried out while PKM was a visitor at the Institute for Mathematics and its Application, University of Minnesota, MN, USA.

\section{Appendix A. Equation of leaf shapes}

We assume that the outline of a leaf is described by the following mathematical function in two dimensional co-ordinates $(x, y)$ :

$y= \pm x^{p}\left(\beta l_{1}-l_{2} x\right)^{q}$

where the exponents $p$ and $q$ are rational numbers, respectively. The parameters $\beta, l_{1}$ and $l_{2}$ are leaf shape parameters. When $p=q=1$, the leaf shape parameters determine the leaf area $S$, leaf lamina length $L$, and leaf lamina width $W$ as follows:

$S=\frac{1}{3} \frac{\left(\beta l_{1}\right)^{3}}{l_{2}^{2}}, L=\frac{\beta l_{1}}{l_{2}}, W=\frac{1}{2} \frac{\left(\beta l_{1}\right)^{2}}{l_{2}}$

\section{Appendix B. Conditions under which light shading occurs}

\section{B.1. Shading from one leaf to another at the same phyllotactic position}

Consider shading on the lower leaf from the upper leaf. The equations of the upper leaf projection on the plane of the lower leaf are:

$y= \pm(x-\xi)\left(\beta l_{1}-l_{2}(x-\xi)\right)$

$\xi=D / \tan (\theta)$

where $\xi$ is the position of the tip of the shadow of the upper leaf measured from the stem (the origin of the $x$ co-ordinate); $\beta$ is a modification parameter of leaf lamina length (see Eq. (A1) and Eq. (A2)); $D$ and $\theta$ are the internodal length of the stem and the elevation angle of the sun measured from the plant (Figs. 1 and 2), respectively. The equation for the lower leaf is:

$y= \pm x\left(l_{1}-l_{2} x\right)$

B.1.1. The elevation angles of the sun for shading to occur/end

The elevation angle of the sun, $\theta_{1}$, at which shading starts is:

$\theta_{1}=\arctan \left(\frac{D l_{2}}{l_{1}}\right)$.

There exist two elevation angles of the sun, $\theta_{2}$ and $\theta_{3}\left(\theta_{2}<\theta_{3}\right)$, such that for any elevation angle of the sun $(\theta)$ between $\theta_{2}$ and $\theta_{3}$ (i.e. $\theta_{2} \leq \theta \leq \theta_{3}$ ) the shaded area is equal to the surface area of the upper (if $\beta<1$ ) or lower leaf (if $\beta>1$ ):

If $\beta$ (shaded area is equal to the surface area of the upper leaf), then

$\theta_{2}=\arctan \left(\frac{D l_{2}}{l_{1}(1-\beta)}\right), \quad$ and $\quad \theta_{3}=\frac{\pi}{2}$.

If $\beta>1$ (shaded area is equal to the surface area of the lower leaf), then

$\theta_{2}=\frac{\pi}{2}, \quad$ and $\quad \theta_{3}=\pi-\arctan \left(\frac{D l_{2}}{l_{1}(\beta-1)}\right)$.

The elevation angle of the sun, $\theta_{4}$, at which shading ends is:

$\theta_{4}=\pi-\arctan \left(\frac{D l_{2}}{\beta l_{1}}\right)$

If the elevation angle of the sun $\theta$ satisfies, $\theta_{1} \leq \theta \leq \theta_{2}$ then the shaded area $S_{\theta}^{\prime}$ at $\theta$ can be calculated by:

$$
\begin{aligned}
S_{\theta}^{\prime}= & 2 \int_{\xi}^{x_{\mathrm{in}}}(x-\xi)\left\{\beta l_{1}-l_{2}(x-\xi)\right\} \mathrm{d} x \\
& +\int_{x_{\mathrm{in}}}^{\frac{l_{1}}{l_{2}}} x\left(l_{1}-l_{2} x\right) \mathrm{d} x
\end{aligned}
$$

where

$x_{\text {in }}=\frac{\xi(\xi+\beta)}{2 \xi+\beta-1}$ 
If $\theta_{2} \leq \theta \leq \theta_{3}$, then according to Eq. (A2) we have

$S_{\theta}^{\prime}=\frac{1}{3} \frac{\left(\beta l_{1}\right)^{3}}{l_{2}^{2}} \quad($ for $\beta<1)$,

or

$S_{\theta}^{\prime}=\frac{1}{3} \frac{l_{1}^{3}}{l_{2}^{2}} \quad($ for $\beta>1)$.

If $\theta_{3} \leq \theta \leq \theta_{4}$, then the shaded area $S_{\theta}^{\prime}$ at $\theta$ can be calculated by:

$$
\begin{aligned}
S_{\theta}^{\prime}= & 2\left[\int_{0}^{x_{\text {in }}} x\left(l_{1}-l_{2} x\right) \mathrm{d} x\right. \\
& \left.+\int_{x_{\text {in }}}^{\xi+\frac{\beta l_{1}}{l_{2}}}(x-\xi)\left\{\beta l_{1}-l_{2}(x-\xi)\right\} \mathrm{d} x\right] .
\end{aligned}
$$

\section{B.2. Shading from upper leaves of neighbouring} plants

By extending the previous case in Section B.1 and considering the shading from an upper leaf of another plant at the same phyllotactic position, the shaded area can be calculated in a similar way. We consider three plants (a), (b), (c), which stand at regular intervals $H$ (from left to right) on a straight line (Fig. 3). We assume the position of the middle stem (b) to be the origin of the $x$ co-ordinate. The equation for describing the leaf projection onto the lower leaf of the middle plant (b) remains the same as Eq. (B1), except for the value of $\xi$. The position of the tip of the shadow of plant (a) on the plane of the lower leaf of plant (b) is expressed by $\xi_{\mathrm{a}}$, and the position of plant (c) by $\xi_{\mathrm{c}}$, that is,

$\xi_{\mathrm{a}}=-H+\frac{D}{\tan (\theta)}$, and $\xi_{\mathrm{c}}=H+\frac{D}{\tan (\theta)}$.

The corresponding position on plant (b), $\xi_{\mathrm{b}}$, is exactly the same as $\xi_{\mathrm{c}}$ in Eq. (B1).

\section{B.2.1. The elevation angles of the sun for shading to occur/end}

The elevation angles of the sun at which shading starts are:

$\theta_{1 \mathrm{a}}=\arctan \left(\frac{D l_{2}}{H l_{2}+l_{1}}\right), \quad$ and $\theta_{1 \mathrm{c}}=\pi-\arctan \left(\frac{\mathrm{Dl}}{\mathrm{Hl}_{2}-l_{1}}\right)$

for plants (a) and (c), respectively.

There exists two elevation angles of the sun for each of plants (a) and (c) $\left(\theta_{2 \mathrm{a}}\right.$ and $\theta_{3 \mathrm{a}}$ for plant (a); $\theta_{2 \mathrm{c}}$ and $\theta_{3 \mathrm{c}}$ for plant (c)) such that for any elevation angle of the sun between the two angles shaded area is equal to the surface area of the upper (if $\beta<1$ ) or lower leaf (if $\beta>1$ ) as in the case of B.1: If $\beta<1$ (shaded area is equal to the surface area of the upper leaf), then,

$\theta_{2 \mathrm{a}}=\arctan \left(\frac{D l_{2}}{H l_{2}+l_{1}(1-\beta)}\right), \quad$ and

$\theta_{3 \mathrm{a}}=\arctan \left(\frac{D}{H}\right)$;

$\theta_{2 \mathrm{c}}=\pi-\arctan \left(\frac{D l_{2}}{H l_{2}+l_{1}(\beta-1)}\right), \quad$ and

$\theta_{3 \mathrm{c}}=\pi-\arctan \left(\frac{D}{H}\right)$.

If $\beta>1$ (shaded area is equal to the surface area of the lower leaf), then

$\theta_{2 \mathrm{a}}=\arctan \left(\frac{D}{H}\right), \theta_{3 \mathrm{a}}=\arctan \left(\frac{D l_{2}}{H l_{2}+l_{1}(1-\beta)}\right) ;$

$\theta_{2 \mathrm{c}}=\pi-\arctan \left(\frac{D}{H}\right)$,

$\theta_{3 \mathrm{c}}=\pi-\arctan \left(\frac{D l_{2}}{H l_{2}+l_{1}(\beta-1)}\right)$.

The elevation angles of the sun at which shading ends are:

$\theta_{4 \mathrm{a}}=\arctan \left(\frac{D l_{2}}{H l_{2}-\beta l_{1}}\right), \quad$ and

$\theta_{4 \mathrm{c}}=\pi-\arctan \left(\frac{D l_{2}}{H l_{2}+\beta l_{1}}\right)$,

for plants (a) and (c), respectively.

\section{References}

Aphalo, P.J., Ballare, C.L., 1995. On the importance of information-acquiring systems in plant-plant interactions. Funct. Ecol. 9, 5-14.

Ballaré, C.L., Sánchez, R.A., Scopel, A.L., Ghersa, C.M., 
1988. Morphological responses of Datura ferox L. seedlings to the presence of neighbours: their relationships with canopy microclimate. Oecologia 76, 288-293.

Ballaré, C.L., Scopel, A.L., Sánchez, R.A., 1990. Far-red radiation reflected from adjaent leaves: an early signal of competition in plant canopies. Science 247, 329332.

Dale, M.P., Causton, D.R., 1992. The ecophysiology of Veronica chamaedrys, $V$. montana and $V$. officinalis: I light quality and light quantity. J. Ecol. 80, 483-492.

Davis, M.H., Simmons, S.R., 1994. Far-red reflected from neighbouring vegetation promotes shoot elongation and accelerates flowering in spring barley plants. Plant Cell Environ. 17, 829-836.

Givnish, T.J., 1982. On the adaptive significance of leaf height in forest herbs. Am. Nat. 120, 353-381.

Givnish, T.J., 1986. Biomechanical constraints on crown geometry in forest herbs. In: Givnish, T.J. (Ed.), On the Economy of Plant Form and Function. Cambridge University Press, Cambridge, pp. 525-583.

Håkanson, L., 1995. Optimal size of predictive models. Ecol. Model. 78, 195-204.

Hara, T., 1984. Dynamics of stand structure in plant monocultures. J. Theor. Biol. 110, 223-239.

Holmes, M.G., Smith, H., 1975. The function of phytochrome in plants growing in natural environment. Nature 254, 512-514.

Huber, H., 1997. Architectural plasticity of stoloniferous and erect herbs in response to light climate, $\mathrm{Ph}$. D. thesis, Utrecht University.

Iwasa, Y., Cohen, D., Leon, J.A., 1984. Tree height and crown shape, as results of competitive games. J. Theor. Biol. 112, $279-297$.

Jørgensen, S.E., 1997a. Ecological modelling in 100 volumes. Ecol. Model. 100, 1-4.

Jørgensen, S.E., 1997b. Ecological modelling by 'Ecological Modelling'. Ecol. Model. 100, 5-10.

Kobayashi, S., 1975. Growth analysis of plant as an assemblage of internodal segments-a case of sunflower plants in pure stands. Jpn. J. Ecol. 25, 61-70.

Morgan, D.C., Smith, H., 1976. Linear relationship between phytochrome photoequilibrium and growth in plants under simulated natural radiation. Nature 262, 210-212.

Müller, F., 1997. State-of-the-art in ecosystem theory. Ecol. Model. 100, 135-161.
Sakai, S., 1991. A model analysis for the adaptive architecture of herbaceous plants. J. Theor. Biol. 148, 535-544.

Sekimura, T., 1993. Phyllotactic patterns of herbaceous plants and solar radiation captured by leaves. Forma 8, 255-261.

Sekimura, T., 1995. The diversity in shoot morphology of herbaceous plants in relation to solar radiation captured by leaves. J. Theor. Biol. 177, 289-297.

Sekimura, T., 1997. The plasticity in shoot morphology of herbs in relation to light capture by leaves. Forma 12, $143-152$.

Sekiyama, F., Kuroiwa, S., Hara, T., 1984a. Relation between yield and planting density in soybean populations. Rep. Soc. Crop Sci. Breed Kinki 29, 42-45.

Sekiyama, F., Kuroiwa, S., Hara, T., 1984b. Relation between yield and planting density in two soybean varieties. Rep. Soc. Crop Sci. Breed Kinki 29, 60-63.

Smith, H., 1982. Light quality, photoreception and plant strategy. Ann. Rev. Plant Physiol. 33, 481-518.

Smith, H., 1994. Sensing the light environment: the functions of the phytochrome family. In: Kendrick, R.E., Kronenberg, G.H.M. (Eds.), Photomorphogenesis, 2nd. Kluwer Academic Publishers, The Hague, pp. 374-416.

Smith, H., 1995. Physiological and ecological function within the phytochrome family. Ann. Rev. Plant Physiol. Plant Mol. Biol. 46, 289-315.

Schmitt, J., Wulff, R.D., 1993. Light spectral quality, phytochrome and plant competition. Trends in Ecol. Evol. 8, $47-50$.

Straškraba, M., 1994. Ecotechnological models for reservoir water quality management. Ecol. Model. 74, 1-38.

Takenaka, A., Satake, K., 1991. Wave-like protonemal colonies of the moth Scopelophila cataractae and their consequences in light capture. J. Bryol. 16, 429-436.

Thompson, L., Harper, J.L., 1988. The effects of grasses on the quality of transmitted radiation and its influence on the growth of white clover Trifolium repens. Oecologia 75, $343-347$.

Tiwari, S., Ramaswamy, R., Rao, J.S., 1996. Adaptive control in a resource management model. Ecol. Model. 84, 53-62.

Van der Molen, D.T., Pintér, J., 1993. Environmental model calibration under different specifications: an application to the model SED. Ecol. Model. 68, 1-19.

Williams, B., 1996. Adaptive optimization of renewable natural resources: solution algorithms and a computer program. Ecol. Model. 93, 101-111. 